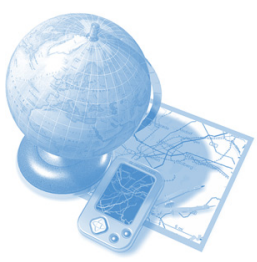

David J McGarvey

School of Physical and

Geographical Sciences

Keele University

Keele

Staffs ST5 5BG

d.j.mcgarvey@

chem.keele.ac.uk

The use of real-world contexts has the potential to enhance the scope of a practical in terms of what students can learn and do

\section{Industry-Linked Context-Based Chemistry Practicals}

\begin{abstract}
There is considerable evidence that the use of tangible contexts enriches the learning experience for students. In view of this, the author has developed two 'industry-linked context-based chemistry practicals' that illustrate the importance of core chemistry topics within commercial/industrial contexts. A common feature of the practicals is that the students work with actual commercial samples and compare their data with that published by the two companies. The principal features of the two practicals are described and contrasted with conventional practicals. Finally, the results of a student evaluation of the practicals are reported.
\end{abstract}

1. 1st Order Kinetics and Photochromism

This practical employs a number of commercial photochromic dyes from James Robinson Ltd (http://www.photochromics.co.uk/index.htm). These colourless dyes become coloured when exposed to UV light and the colour fades thermally when the UV light source is removed. The observed colour and kinetic behaviour of the dyes are sensitive to the molecular structure, environment and temperature. The dyes have applications in ophthalmic lenses, security applications and novelty items. The experiment involves measurement of UV-VIS spectra of the colourless and coloured forms of the dyes and the dye fading kinetics. The students are able to compare their kinetic and spectroscopic data with data published by James Robinson on their website.

2. An Investigation of the UV Absorbing Properties of Commercial Organic Sunscreens This practical employs three commercial organic sunscreens from DSM Nutritional Products (http://www.dsm.com/en US/html/dnp/personal suncare.htm) and involves measurements of UV absorption spectra, molar absorption coefficients and the investigation of spectral changes resulting from exposure of the sunscreens to UV light. In addition to learning about the Beer-Lambert law and UV spectrophotometry, students also learn about the mechanisms by which organic sunscreens operate, their molecular structures, industry-specific jargon and units, and the biological effects of exposure to different types of UV radiation. Students compare their measurements with data published by DSM. An additional feature of the practical is the use of an on-line sunscreen simulator provided by CIBA (https://www.cibasc.com/

pccibasunscreensimulator/), which requires registration before use. Students formulate their own suncreams on-line and save the results as a pdf file. They then have to extract information from the data sheet in order to determine the UVA protection factor (Boots star rating).

\section{Introduction}

There is considerable evidence that the use of tangible contexts enriches the learning experience for students ${ }^{1-4}$. In view of this, I recently developed two 'industry-linked context-based chemistry practicals', each of which focuses on core chemistry topics within commercial or industrial contexts. A common feature of the practicals is that the students work with actual commercial samples and compare their experimental data with that published by the companies. The principal features of the two practicals are described and future developments to the practicals are discussed in the light of student feedback and my own observations.

\section{1. $1^{\text {st }}$ Order Kinetics and Photochromism}

A context-based chemistry practical highlighting the pivotal role of kinetics in commercial applications of organic photochromic dyes.

Sunglasses that darken in the sunlight and revert to near colourless in subdued light are familiar to most of us and constitute the most widely known application of the phenomenon known as photochromism ${ }^{5}$. What is not so familiar however is the pivotal 
role that chemistry plays in this fascinating and often spectacular phenomenon, and also the diverse range of applications and potential applications that exist beyond the ophthalmic lens market (eg fuel markers, security inks and packaging, cosmetics and fashion, electronic nanotechnology $)^{6-8}$.

Although the first photochromic sunglasses used silver halide technology, more recent developments have focused on organic photochromic dyes, particularly spiro-naphthoxazines and naphthopyrans (Figure 1). James Robinson ${ }^{6-8}$, based in Huddersfield, has been actively involved in the development of such molecules since 1990 and currently markets a range of photochromic dyes under the Reversacol trade name (http:// www.photochromics.co.uk/index.htm).

This practical employs a number of Reversacol photochromic dyes in toluene solution. The dyes become coloured when exposed to UV light and the colour fades thermally when the UV light source is removed. Spiro-naphthoxazines and naphthopyrans can be visualised as being composed of two planar components held at right angles (Figure 1). The photochromic effect arises from an electrocyclic ring opening that enables the two components to occupy the same plane resulting in extended p-electron de-localisation (increased conjugation) and a consequent shift of the spectral absorption into the visible region. The reverse reaction is usually thermally driven, but in some cases can be driven by visible light, and proceeds at a rate that is dependent on numerous factors including molecular structure, temperature and the molecular environment. Structural modifications such as variation of peripheral substituents enable chemists at James Robinson to fine-tune the spectroscopic (ie colour) and kinetic properties of the dyes according to the requirements of the application. In solution the reverse reaction usually follows first order kinetics, but the kinetic behaviour in other environments such as polymers and plastics can be more complex.

What struck me when I first explored the possibilities of using photochromism as the basis for an undergraduate chemistry practical is the crucial importance of the spectroscopic and kinetic properties of the dyes for applications in the ophthalmic lens market. It is clear that amongst the desirable properties of photochromic dyes for use in ophthalmic lenses, rapid colouration combined with an acceptable fade rate is a high priority. In addition, the temperature dependence of the fade rate, which can affect the depth of colour achieved under constant illumination, should ideally be minimal. Here is an example of a commercial application of synthetic organic chemistry that depends on exercising control over physicochemical properties (kinetics and spectroscopy). Specifically, the importance of fundamental kinetic parameters (half-lives, rate constants, activation energies) as determining factors within a tangible industrial/commercial context was attractive.
The Practical

Undergraduate experiments based on the kinetics and spectroscopy of photochromic dyes are not new (see for example, Prypsztejn and Negri $\left.{ }^{9}\right)$. However, I thought that such
Spiro-naphthoxazines
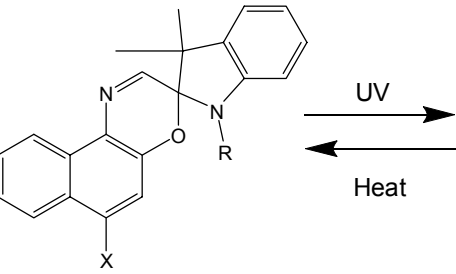

Figure 1: Generic structures of the colourless and coloured forms of spironaphthoxazine and naphthopyran photochromic dyes. Adapted from reference 7. a practical could be significantly enhanced if it involved the students working with actual commercial dyes. Inspired by a Chemistry in Britain article ${ }^{6}$, I contacted James Robinson who kindly supplied samples of some of the Reversacol photochromic dyes in addition to valuable feedback on the proposed practical.

An advantage of using commercial samples is that a wealth of information resources are accessible via the James Robinson website (http://www.photochromics.co.uk/index.htm) and this facility thus enables students to gain some insight into the chemistry and applications of photochromism as well as comparing their experimental data with the data published by James Robinson.

What does the practical involve?

The practical shows how an understanding of 1st order kinetics and spectrophotometry are important within an industrial context and also how this understanding aids interpretation of technical product data. It is used in the first year chemistry course at Keele University, but is highly flexible and easily adaptable to suit students at other levels. 
Some of the chemical topics and principles that the practical highlights include:

- The relationship between spectral absorption profile and colour.

- Analysis of $1^{\text {st }}$ order kinetic data.

- Analysis of the temperature dependence of rate constants.

- Influence of changes in p-electron de-localisation on absorption spectra.

In its present form the practical involves:

- Acquisition of UV-VIS absorption spectra of the colourless and coloured forms of the dyes.

- Analysis of first order kinetic data (Measurement of the rate constants and half-lives for the fading of the coloured forms of the dyes.

- Investigation of the temperature dependence of the fade rate and measurement of the activation energy for the fading process of one of the dyes.

- Chemical structure drawing.

- Use of online resources available on the James Robinson website.

- Comparison of acquired data with spectroscopic and kinetic data published by James Robinson Ltd.

- Exposure to the variety of chemical structures used for organic photochromics, their spectroscopic and chemical properties and how they work.

- Exposure to some of the applications of photochromism and how kinetic and spectroscopic considerations are important for these.

- Exposure to technical jargon and terminology used in the industry.

\section{Equipment and Materials}

UV-VIS spectrophotometer, flashlight or UVA lamp, quartz cuvettes, thermometer. HPLC grade toluene. Solutions $\left(\sim 10^{-4} \mathrm{~mol} \mathrm{dm}^{-3}\right)$ of various Reversacol photochromic dyes dissolved in HPLC grade toluene.

\section{Results}

Pre-Lab Work: The James Robinson website provides some excellent general information on photochromism as well as a number of informative articles available as pdf files from the 'news' link. Using these resources students are asked to find a definition of 'photochromism' and some information relating to current/potential applications.
UV-VIS Absorption Spectra: For the colourless forms of the dyes this is a straightforward aspect of the practical. However, using a scanning spectrophotometer to record the spectrum of the coloured form of the dye is more challenging, particularly

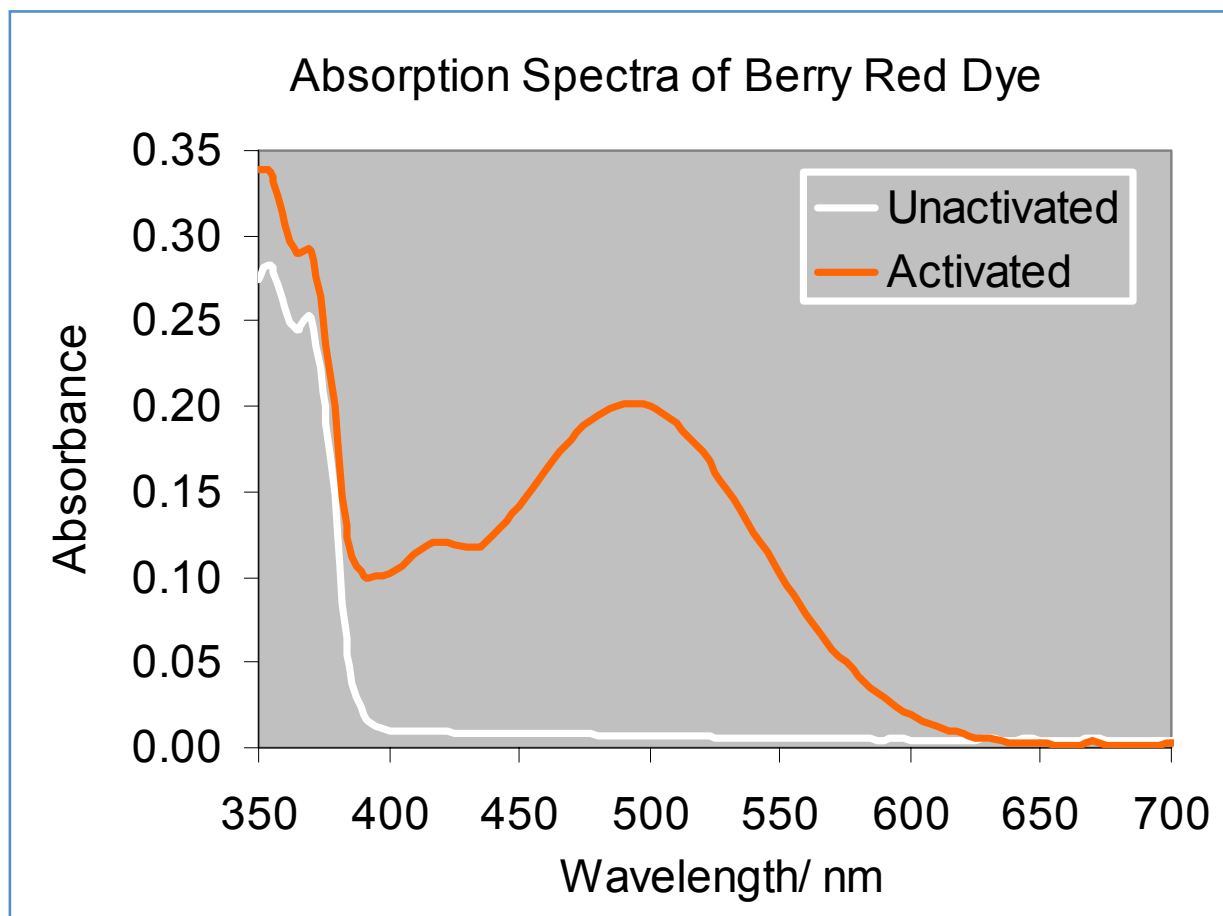

Absorption Spectra of Palatinate Purple Dye

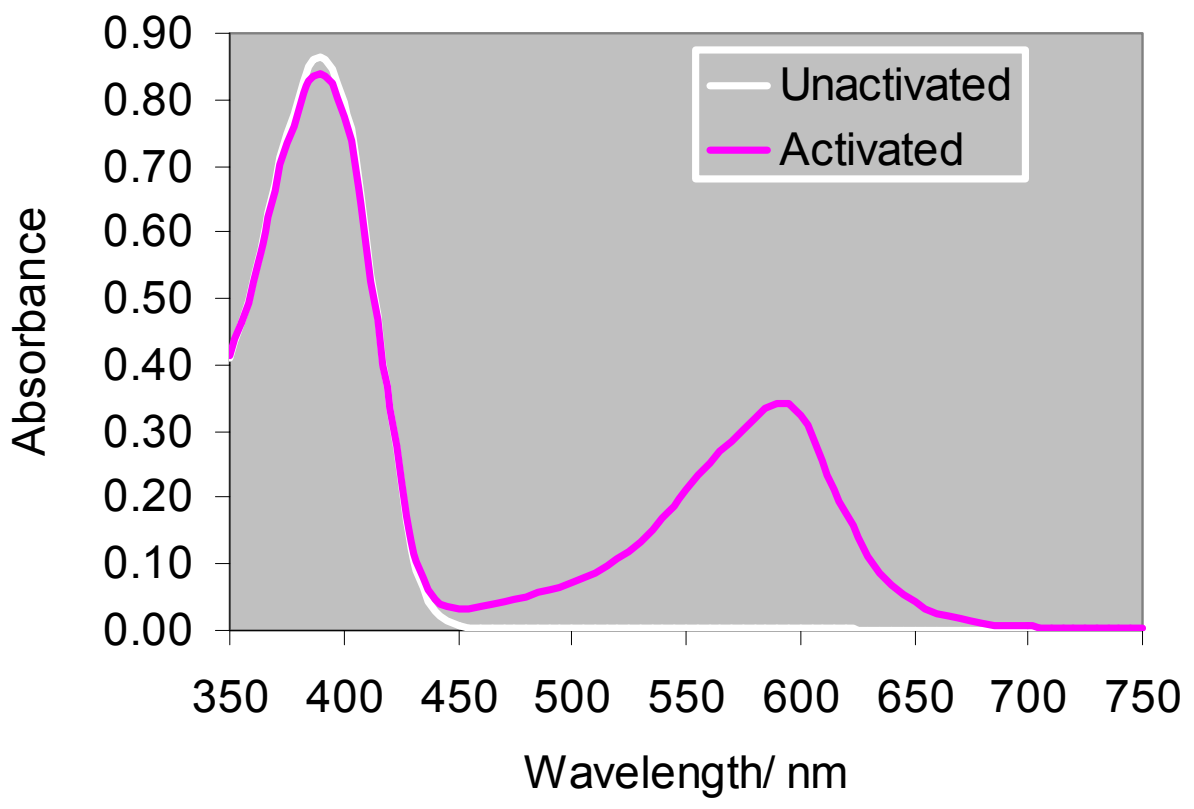

Figure 2: Absorption spectra of Reversacol photochromic dyes $\left(\sim 10^{-4} \mathrm{M}\right.$ in toluene).

for dyes with short half-lives (the half-lives vary from $\sim 10 \mathrm{~s}$ to $>100 \mathrm{~s}$ at room temperature) as the dye fades significantly as the spectrum is being acquired. This can cause an apparent shift in the position of the absorption maximum $\left(I_{\max }\right)$ when compared with the James Robinson data, but the shift is small (a few $\mathrm{nm}$ ). It is also important to achieve uniform colouration 
of the solution throughout the cuvette, which is best achieved by stirring during or immediately after illumination. The solutions are effectively 'coloured up' using a handheld UVA lamp or flashlamp. Students compare their $I_{\max }$ values with those published by James Robinson on their website. Representative spectra are shown in Figure 2. important to achieve uniform colouration throughout the entire volume of solution within the cuvette. As we do not have sufficient temperature control apparatus for the number of students in the class at Keele, the kinetic measurements are performed at ambient temperature, which is recorded. For the majority of dyes the fade kinetic are $1^{\text {st }}$ order in toluene solution and the analysis of the absorbance versus time curves involves manipulation of the data on a spreadsheet to plot $\ln \left(A-A_{\infty}\right)$ versus $t$, where $A_{\infty}$ is the final absorbance at thermal equilibrium. The slope of the resulting linear plot is $-k$ from which the half-life is easily obtained. Representative data is shown in Figure 3. The students compare their halflives with the values published by James Robinson, noting of course that the James Robinson values pertain to $20^{\circ} \mathrm{C}$. Temperature dependence measurements involve a method based on initial rates.

\section{Student Feedback}

The student feedback on the practical was generally very positive. Representative student comments include:

Photochromism: What did you like about this practical?

- I like the dyes. I haven't seen these kinds of dyes before. It's good to know how chemistry is applied in our lives.

- Very interesting, good experiment to do as is completely new.

- Using the lamps to change the colour of the solution.

- It was interesting and different.

- Something new.

- Interesting and the way the chemicals changed colour under UV.

- The vibrant colours of the dyes.

- Very helpful in understanding lectures - kinetic graphs etc.

- Enjoyable.

Fade Kinetics: Having located the $\lambda_{\max }$ of the coloured forms of each of the dyes students then set the spectrophotometer to this wavelength and record the fall in absorbance with time. Here students have to exercise judgement over the data collection interval and total data collection time. As before, it is
- Saw reaction right in front of us.

- We got to organise our own experiment.

- Experience in using spectrophotometer for rates. 
Photochromism: What did you dislike about this practical?

- I didn't know what I was doing during the experiment.

- It was a bit monotonous after a while.

- Difficult to find information.

- Repetitive.

- Waiting around while data being collected.

- The maths.

Conclusion

From a combination of the student feedback (including anecdotal feedback) and my own observations it is clear that the students find the practical novel and interesting, but that perhaps they are being overloaded with information and being asked to do too much of the same thing. One of the implicit aims of the practical is that students see why kinetic considerations in commercial applications of photochromism are important and I would like to have clearer evidence that the students make such links. This may be best achieved by the design of pre-laboratory and/or post-laboratory activities that require students to apply their knowledge in a commercial context. Future development of the practical will therefore focus on improvements to the design of pre-laboratory and post-laboratory work and a review of the overall information and work load.

\section{An Investigation of the UV Absorbing Properties of Commercial Organic Sunscreens}

A context-based chemistry practical highlighting the pivotal role of UV absorption spectra, photoisomerisation, the BeerLambert law and molar absorption coefficients in commercial sunscreens.

Organic sunscreens function in variety of ways, but a common feature of all organic sunscreens is that they are designed to be highly efficient and photostable UV absorbers that are able to dissipate the absorbed energy rapidly, efficiently and harmlessly as heat ${ }^{11}$. The sunscreens used in this practical achieve this through photo-induced geometrical ( $E-Z$ or cistrans) isomerisation and each displays a strong absorption band that peaks in the UVB region (290-320 nm).

The practical employs three commercial organic sunscreens (Figure 4) from DSM Nutritional Products (http:// www.dsm.com/en_US/html/dnp/personal_suncare.htm) and involves measurements of UV absorption spectra, molar absorption coefficients and investigation of spectral changes resulting from exposure of the sunscreens to UV light. In addition to learning about the Beer-Lambert law and UV spectrophotometry, students also learn about the mechanisms by which organic sunscreens function, their molecular structures, industry-specific jargon and units, and the biological effects of exposure to different types of UV radiation. Students compare their measurements with data published by DSM.

An additional feature of the practical is the use of an on-line sunscreen simulator provided by CIBA (https:// www.cibasc.com/pccibasunscreensimulator/), which requires registration before use. Students formulate their own suncreams on-line and save the results as a pdf file. They then have to extract information from the data sheet in order to determine the UVA protection factor (Boots star rating).
The Practical

UV protection and sunscreens provides a versatile real-life context that is suitable for a wide range of educational activities at various levels (see for example, Abney and Scalettar ${ }^{10}$ ). The design of this practical was largely informed and inspired by my previous research on the photochemistry of sunscreens ${ }^{11-13}$.

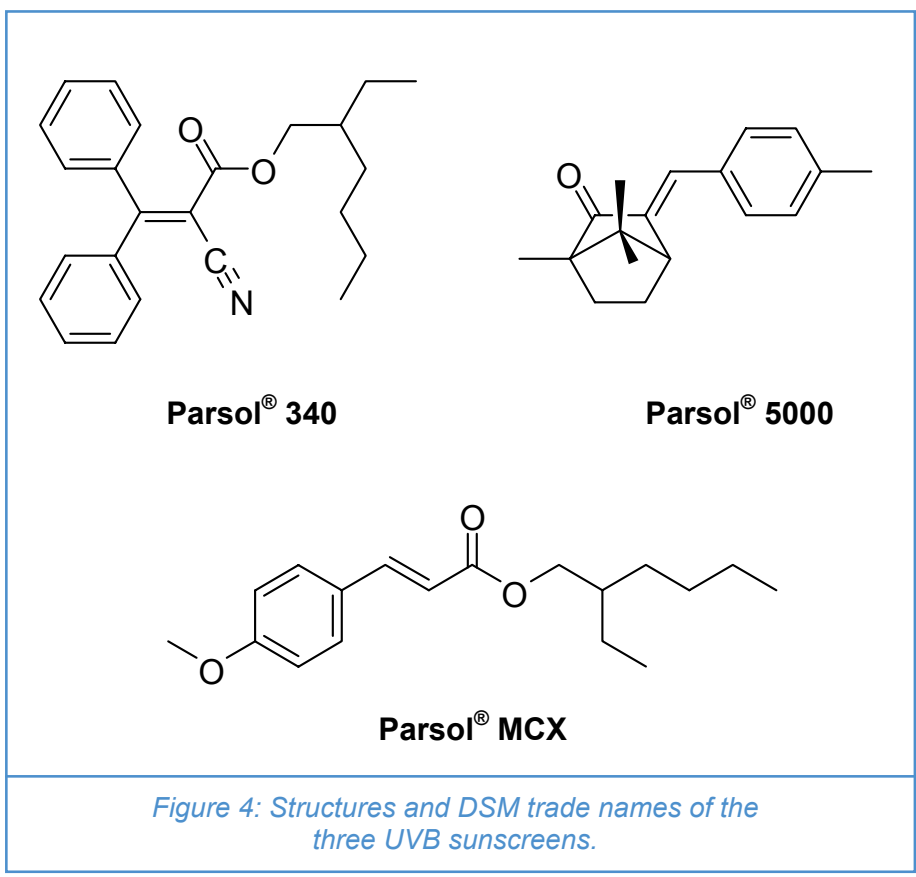

What does the practical involve?

The practical shows how an understanding of the BeerLambert law and UV spectrophotometry are important within a commercial context and also how this understanding aids interpretation of technical product data. It is used in the first year chemistry course at Keele University, but is highly flexible and easily adaptable to suit students at other levels. Some of the chemical topics and principles that the practical highlights include:

- Classification of different UV regions.

- UV absorption spectroscopy.

- Application of the Beer-Lambert law to determine molar absorption coefficients.

- A sense of scale for molar absorption coefficients.

- Inter-conversion of units.

- Photoisomerisation.

- Influence of geometrical isomerisation on absorption spectra.

- 'Photostability' in the context of sunscreens.

In its present form the practical involves:

- Measurement of the UV absorption spectra of the three sunscreens.

- Application of the Beer-Lambert law for the determination of the molar absorption coefficients of the three sunscreens.

- Investigation of any modifications to the UV absorption profile of the sunscreens as a result of inducing photoisomerisation by exposure to UV light

- Use of the Ciba on-line sunscreen simulator to formulate a sunscreen and obtain SPF and UVA protection factor information in addition to spectral data. 


\section{Equipment and Materials}

UV-VIS spectrophotometer, quartz cuvettes, UV (313 nm) light source (at Keele we use foster + freeman VSC4c video spectral comparators from our Forensic Science laboratory) but sunlight can of course be used, if available. HPLC grade methanol and ethanol. Solutions of accurately known concentration $\left(\sim 10^{-4} \mathrm{~mol} \mathrm{dm}^{-3}\right)$ of the three sunscreens dissolved in methanol or ethanol.

\section{Results}

Pre-Lab work: In preparation for the practical the students are required to find out the wavelength regions corresponding to UVA, UVB, UVC, Visible and IR radiation and the relative proportions of these penetrating the atmosphere to sea-level. The students also learn about the differing biological effects of UVA and UVB radiation, the different UV absorption properties of glass and quartz and they also carry out serial dilution calculations.

UV Absorption spectra: The students acquire the absorption spectra of the three sunscreen solutions and compare the $I_{\max }$ values with those available from DSM. The student data is consistent with that reported by DSM to within $\pm 2 \mathrm{~nm}$.

Determination of molar absorption coefficients: The students use autopipettes and/or conventional pipettes to prepare a series of solutions of varying concentration (typically $10^{-5}-10^{-4} \mathrm{M}$ ) by accurate dilution of the stock solutions in 5 $\mathrm{ml}$ or $10 \mathrm{ml}$ volumetric flasks. They then record the absorption spectra of the different solutions and plot absorbance at $I_{\max }$ versus concentration to determine the molar absorption coefficient from the slope. Typical data is shown in Figure 5

DSM report molar absorption coefficients as 'UV specific extinction' values. These values represent the projected absorbance of a $1 \%$ solution of the sunscreen in a cuvette of 1 $\mathrm{cm}$ pathlength. Therefore, to compare their results with DSM students need to convert the units of their own value or the DSM value. Converting the DSM value involves translating the $1 \%$ solution into molarity and then applying the Beer-Lambert law to obtain e in $\mathrm{dm}^{3} \mathrm{~mol}^{-1} \mathrm{~cm}^{-1}$. For Parsol 340, DSM quote a UV specific extinction in the range 340-370, which converts to $12,291-13,375 \mathrm{dm}^{3} \mathrm{~mol}^{-1} \mathrm{~cm}^{-1}$, in good agreement with the slope for the data in Figure 5

Investigation of spectral changes induced by exposure to UV light: Here the students investigate whether spectral changes occur as a result of exposure to UV light. It should be noted that the results for this part of the experiment are somewhat dependent on the properties (eg spectral intensity profile) of the UV light source used, but the effects are apparent using natural sunlight (of course, because glass absorbs UVB you have to open the window, or go outside). At Keele the students expose the sunscreen solutions in quartz cuvettes to $313 \mathrm{~nm}$ UV light from a video spectral comparator for short $(\sim 10 \mathrm{~s})$ intervals and record the absorption spectra after each exposure. Model data is shown in Figure 6.

An important principle of the operation of these types of sunscreens is illustrated by this part of the experiment. Where the two geometrical isomers are different (Parsol 5000, Parsol MCX) then the exposure to UV light leads to the development of a photo-stationary isomer mixture with a resulting modest modification to the UV spectral profile of the solution that reflects the fact that the two isomers have slightly different UV spectra. The precise composition of the photo-stationary mixture is dependent on numerous factors, and not least the properties of the UV light source. The important point is that a photo-stationary mixture is quickly established and it is this mixture that exhibits photostability in that its spectral profile
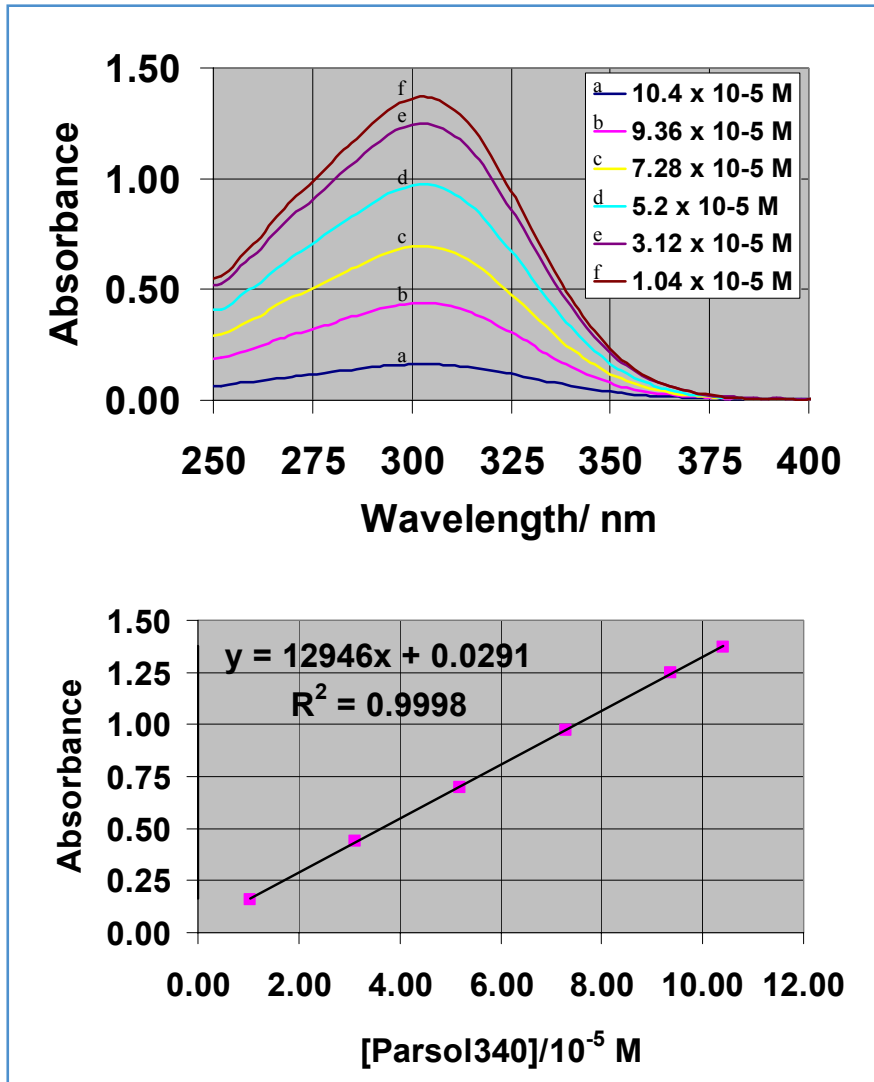

Figure 5: Beer-Lambert law data for Parsol 340 in methanol.

remains constant for extended periods of UV exposure; ie both isomers are effective at absorbing UV and dissipating the energy via photoisomerisation. Parsol 340 does undergo 'photoisomerisation', but of course both 'isomers' are identical and this accounts for the lack of any significant effect on its spectral profile as a result of exposure to UV light. The students learn an important point here - the sunscreen is not 'going off' or 'breaking down'; a photostationary photostable isomer mixture is established and this is what it is designed to do!

Ciba Sunscreen Simulator: Here the students have to register on-line in order to be able to use the simulator. The simulator calculates the sun protections factor (SPF), UVA/UVB ratio and other parameters for a specified formulation of ingredients at specified concentrations. The program returns the spectral absorption and transmission profiles of a model sunscreen film, which the students can relate to the SPF and UVA protection factors, and the results can be saved as a pdf file for inclusion with the laboratory report. At present the students are simply required to formulate their own sunscreen (including at least one of the sunscreens they have studied) and to report the SPF and UVA protection ratings. An example of the output information is given in Figure 7 . 

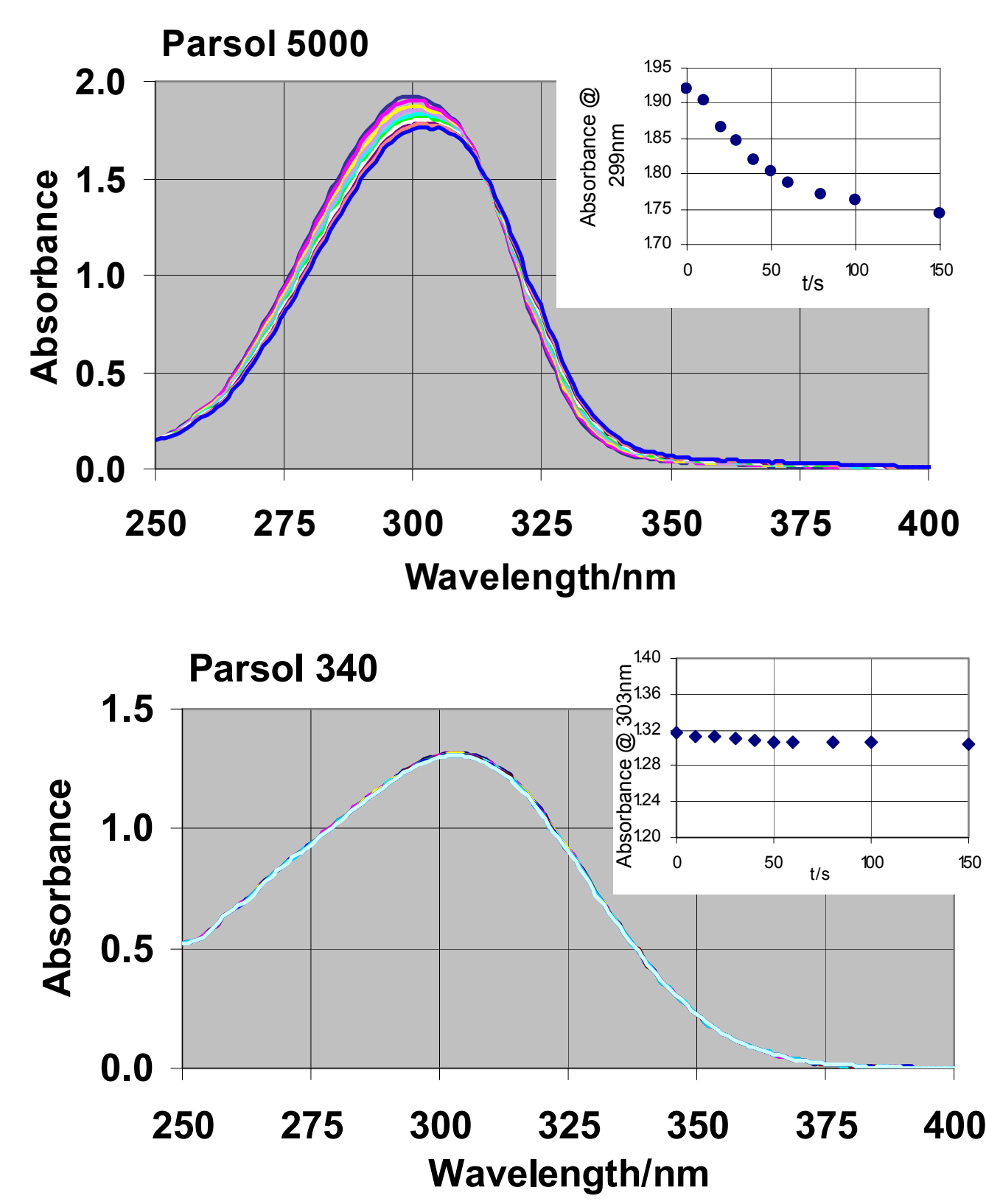

Figure 6: The effects of exposure to $313 \mathrm{~nm}$ UV light on the spectral absorption profiles of Parsol 5000 and Parsol 340. For Parsol 5000, the photo-stationary and photostable mixture is established by $150 \mathrm{~s}$.
- As it dealt with a commercial product, I felt like it wasn't one of those purposeless experiments.

- Useful in future teamwork.

The following comments were obtained from mature students who did the practical as part of a prePGCE subject enhancement course:

- An excellent 'real life' exercise. Good practice of basic lab techniques and reinforcement of practical spectroscopy. Excellent supporting paperwork made it relatively straightforward to carry out and the requirements were well defined.

- Very relevant given the time of year! Lots of interesting data emerged which generated much thought on the issue of sun protection.

Sunscreens: What did you dislike about this practical?

- Too long.

- In a group, some just stand and watch.

- Not sure what I was doing.

- Repetitive.

- Write-up very time consuming.

- Got confused

- Boring.

- Not enough time to repeat measurements.

\section{Student Feedback}

The student feedback on the practical was generally very positive with negative comments largely relating to the workload associated with the practical and the write-up. Representative student comments include:

Sunscreens: What did you like about this practical?

- Learning to use a spectrophotometer.

- Interesting to see the effect of sunscreens.

- Creating my own sunscreen.

- Effects of UVA, UVB interesting.

- Interesting practical.

- Learning how to use autopipettes.

- Interesting and fun to research.

- Interesting to learn about the chemical properties and structures of sunscreens.

\section{Conclusion}

From a combination of the student feedback (including anecdotal feedback) and my own observations it is clear that the students generally enjoyed the practical, found it interesting and a good learning experience. In addition, it is evident that the real-life context of the practical was received positively. However, as with the photochromism practical, it is evident that some students feel that the practical is too long and a bit repetitive, and that the write-up is very timeconsuming. On reflection, it is evident that the demands of the experimental work and the write-up can be significantly reduced whilst still achieving the desired learning outcomes. Future development of the practical will therefore focus on considered modifications to the design of pre-laboratory and post-laboratory work and a review of the overall information load and student workload. 


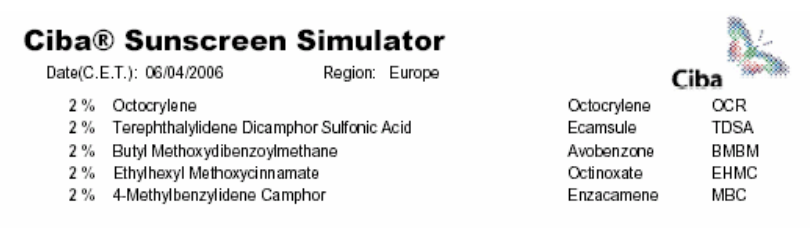

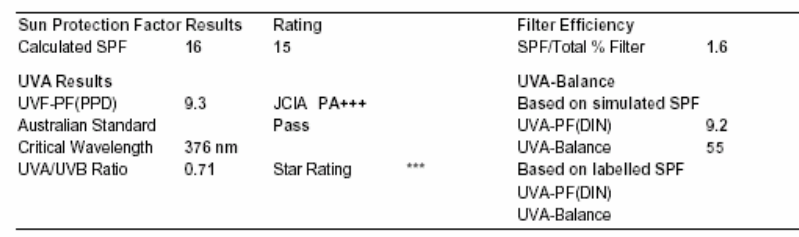

Model Film Extinction

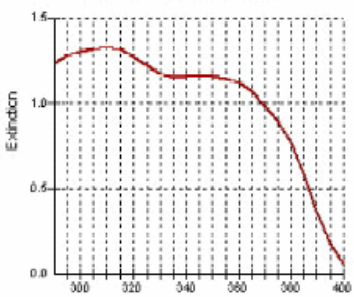

wavelenght $/ \mathrm{nm}$

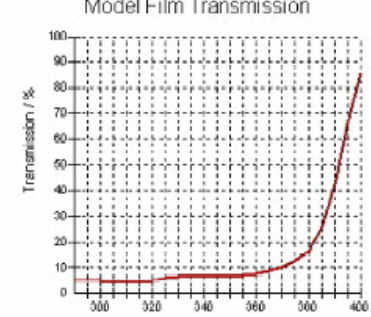

Wrovelenght $/ \mathrm{nm}$

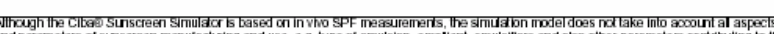

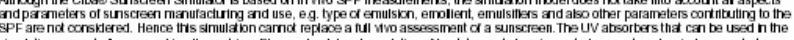

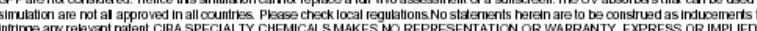

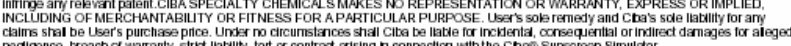

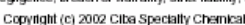

Figure 7: Ciba sunscreen simulator example (https://www.cibasc.com/pccibasunscreensimulator/)

\section{Summary}

The use of real-world contexts has the potential to enhance the scope of a practical in terms of what students can learn and do. In this article I have described the principal features of what I have termed industry-linked practicals. The distinctive features of the practicals can be summarised as:

- Core chemistry within tangible industrial/commercial contexts.

- Handling of actual commercial samples.

- Practical activities that mirror those undertaken in industry.

- Information retrieval using on-line resources (pre-lab and/ or post-lab).

- Exposure to, and interpretation of, industry-specific data, terminology and jargon.

- Acquisition of experimental data that can be directly (or indirectly) compared with available commercial technical data.

- Learning about the chemistry behind commercial products and some of the key issues for the design and marketing of these products.

The practicals have generally been received positively by students and it is evident that they find the contexts interesting and enjoyable. However, the demands placed on students in terms of acquisition and analysis of data and assimilating information is perhaps excessive and further development of the practicals will aim to address these issues.
In addition, there is more scope within each practical to provide more opportunities for students to apply their knowledge within specific commercial contexts.

\section{Acknowledgements}

The author is grateful to Helen Parry, Business Manager at James Robinson, Huddersfield for supplying the Reversacol photochromic dyes and for helpful discussions. The author also thanks Ulrich Huber of DSM Nutritional products for helpful discussions and for supplying the Parsol sunscreen samples and Bernd Herzog of Ciba Specialty Chemicals Inc. for useful information relating to the Ciba sunscreen simulator. I would also like to thank $1^{\text {st }}$ year (2005-2006) Keele University Chemistry/Medicinal Chemistry undergraduates and students (2005-2006) on the Keele TTA Chemistry Enhancement Course for their valuable feedback. Finally, I would like to thank The Higher Education Academy Physical Sciences Subject Centre for funding of a Development Project (2004).

\section{References}

1. Belt, Simon T., Leisvik, Mathias J., Hyde, Andrew J. and Overton, Tina L., Using a context-based approach to undergraduate chemistry teaching - a case study for introductory physical chemistry, Chem. Educ. Res. Prac., 6 (3) 166-179 (2005)

2. Belt, Simon T. and Kendall, Sue B., Educ. Chem., 43(1) 15-17 (2006)

3. Tsaparlis, G. and Gorezi, M., A modification of a conventional expository physical chemistry laboratory to accommodate an inquiry/project-based component: method and students' evaluation, Canadian Journal of Science, Mathematics and Technology Education, 5, 111-131 (2005)

4. http://www.planet-science.com/sciteach/review/ Findings.pdf

5. Bouas-Laurent, $H$. and Dürr, $H$., Organic Photochromism (IUPAC Technical Report), Pure Appl. Chem., 73, (4) 639-665 (2001)

6. Higgins, S., Chasing a Rainbow, Chemistry in Britain, 39 (6), 26-29, (June 2003)

7. Higgins, S., Photochromics - Colourful Molecular Atheletes, Chemistry Today, (January-February 2003) available as a pdf file at http:// www.photochromics.co.uk/index.htm

8. Parry, H., Corns, N. and Towns, A., New Uses for Photochromics, Speciality Chemical Magazine (October 2003) - available as a pdf file at http:// www.photochromics.co.uk/index.htm

9. Prypsztejn, H. E. and Negri, R. M., J. Chem. Ed. 78, 645-648 (2001)

10. Abney, J. R. and Scalettar, B. A., J. Chem. Ed. 75, 757760 (1998)

11. Cantrell, A., McGarvey, D. J. and Truscott, T. G., Photochemical and photophysical properties of sunscreens, in Sun Protection in Man, Comprehensive Series in Photosciences, Chapter 26, 495-519, Volume 3, Edited by Giacomoni, P. U., Elsevier (2001)

12. Cantrell, A. and McGarvey, D. J., J. Photochem. Photobiol. B, 64 (2-3) 117-122 (2001)

13. Cantrell, A., McGarvey, D. J., Mulroy, L. and Truscott, T. G., Photochem Photobiol 70 (3) 292-297 (1999) 